\title{
Properties of Heavy Secondary Fluorine Cosmic Rays Results from the Alpha Magnetic Spectrometer
}

\author{
Qi Yan ${ }^{a, *}$ and Vitaly Choutko ${ }^{a}$ \\ ${ }^{a}$ Massachusetts Institute of Technology (MIT), Cambridge, MA 02139, USA \\ E-mail: qyan@cern.ch
}

Precise knowledge of the charge and rigidity dependence of the secondary cosmic ray fluxes and the secondary to primary flux ratios is essential in the understanding of cosmic ray propagation. We report the properties of secondary cosmic ray fluorine $\mathrm{F}$ in the rigidity $R$ range $2.15 \mathrm{GV}$ to 2.9 TV based on 0.29 million events collected by the Alpha Magnetic Spectrometer experiment on the International Space Station. The fluorine spectrum deviates from a single power law above $200 \mathrm{GV}$. The heavier secondary-to-primary F/Si flux ratio rigidity dependence is distinctly different from the lighter $\mathrm{B} / \mathrm{O}$ (or B/C) rigidity dependence. In particular, above $10 \mathrm{GV}$, the $\frac{\mathrm{F} / \mathrm{Si}}{\mathrm{B} / \mathrm{O}}$ ratio can be described by a power law $R^{\delta}$ with $\delta=0.052 \pm 0.007$. This shows that the propagation properties of heavy cosmic rays, from $\mathrm{F}$ to $\mathrm{Si}$, are different from those of light cosmic rays, from He to $\mathrm{O}$.

$37^{\text {th }}$ International Cosmic Ray Conference (ICRC 2021)

July 12th-23rd, 2021

Online - Berlin, Germany

\footnotetext{
${ }^{*}$ Presenter
} 


\section{Introduction}

Fluorine nuclei in cosmic rays are thought to be produced mostly by the collisions of heavy nuclei, such as $\mathrm{Ne}, \mathrm{Mg}$, and $\mathrm{Si}$, with the interstellar medium. Together with the much more abundant $\mathrm{Li}, \mathrm{Be}$, and $\mathrm{B}$ cosmic rays, they are called secondary cosmic rays [1]. Fluorine is the only pure secondary cosmic ray between oxygen and silicon [2].

The secondary-to-primary flux ratios of light nuclei in cosmic rays, in particular B/C or the more direct $\mathrm{B} / \mathrm{O}$, have been traditionally used to study the propagation of cosmic rays in the Galaxy [8]. In previous publications, AMS has shown that all light secondary-to-primary ratios, $\mathrm{Li} / \mathrm{C}, \mathrm{Li} / \mathrm{O}, \mathrm{Be} / \mathrm{C}$, $\mathrm{Be} / \mathrm{O}, \mathrm{B} / \mathrm{C}$ and $\mathrm{B} / \mathrm{O}$, deviate from a single power law (harden) above $200 \mathrm{GV}[9,10]$. Recently, AMS also has studied the properties of the primary heavy $\mathrm{Ne}, \mathrm{Mg}$, and $\mathrm{Si}$ fluxes [11] and found that they form a separate class of primary cosmic rays. Differences in the rigidity dependence of the $\mathrm{F}$ flux and light secondary cosmic ray $\mathrm{Li}, \mathrm{Be}$, and $\mathrm{B}$ fluxes, as well as differences in the rigidity dependence of light $(\mathrm{B} / \mathrm{O})$ and heavy $(\mathrm{F} / \mathrm{Si})$ secondary-to-primary flux ratios, provide new important insights on cosmic ray propagation.

We report the precise measurement of the F flux in the rigidity range from $2.15 \mathrm{GV}$ to $2.9 \mathrm{TV}$ based on 0.29 million fluorine nuclei collected by AMS during the first 8.5 years (May 19, 2011 to October 30, 2019) of operation aboard the International Space Station (ISS). The total flux error is $5.9 \%$ at $100 \mathrm{GV}$.

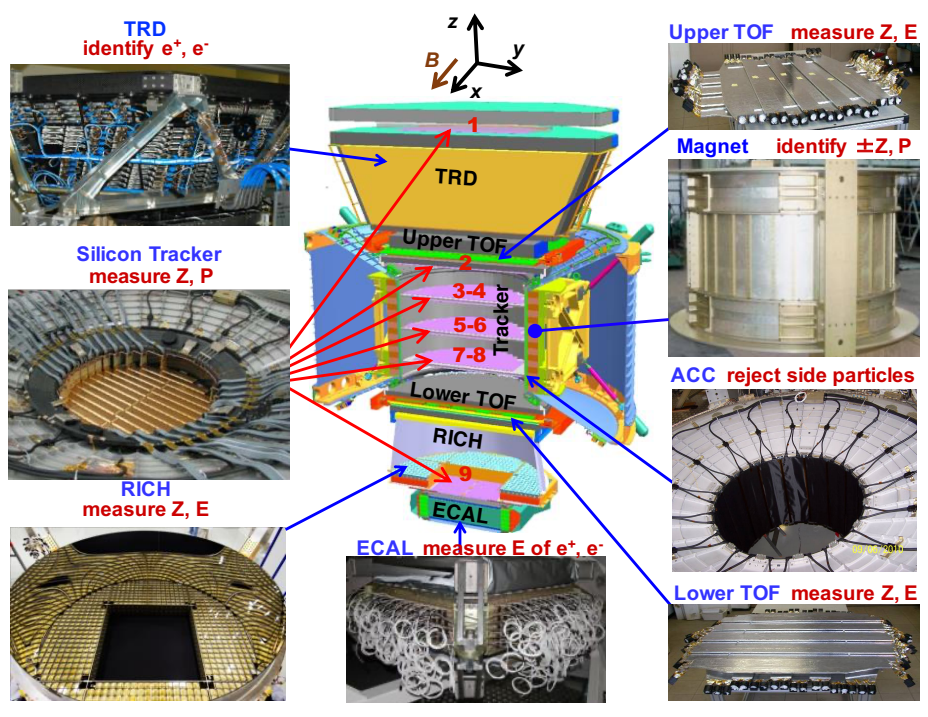

Figure 1: The AMS detector and its main elements.

\section{AMS Detector}

AMS is a general purpose high energy particle physics detector in space [12]. The layout of the detector is shown in Fig. 1. The key elements used in this measurement are the permanent magnet, the nine layers, $L 1-L 9$, of silicon tracker [13-15] and the four planes of time of flight TOF scintillation counters [16]. Further information on the AMS layout and performance is detailed in Ref. [10]. 


\section{Event Selection}

Fluorine events are required to be downward going and to have a reconstructed track in the inner tracker which passes through $L 1$. In the highest rigidity region, $R \geq 1.2 \mathrm{TV}$, the track is also required to pass through $L 9$. Track fitting quality criteria such as a $\chi^{2} /$ d.o.f. $<10$ in the bending coordinate are applied.

The measured rigidity is required to be greater than a factor of 1.2 times the maximum geomagnetic cutoff within the AMS field of view. The cutoff was calculated by backtracing particles from the top of AMS out to 50 Earth's radii using the most recent IGRF model [19].

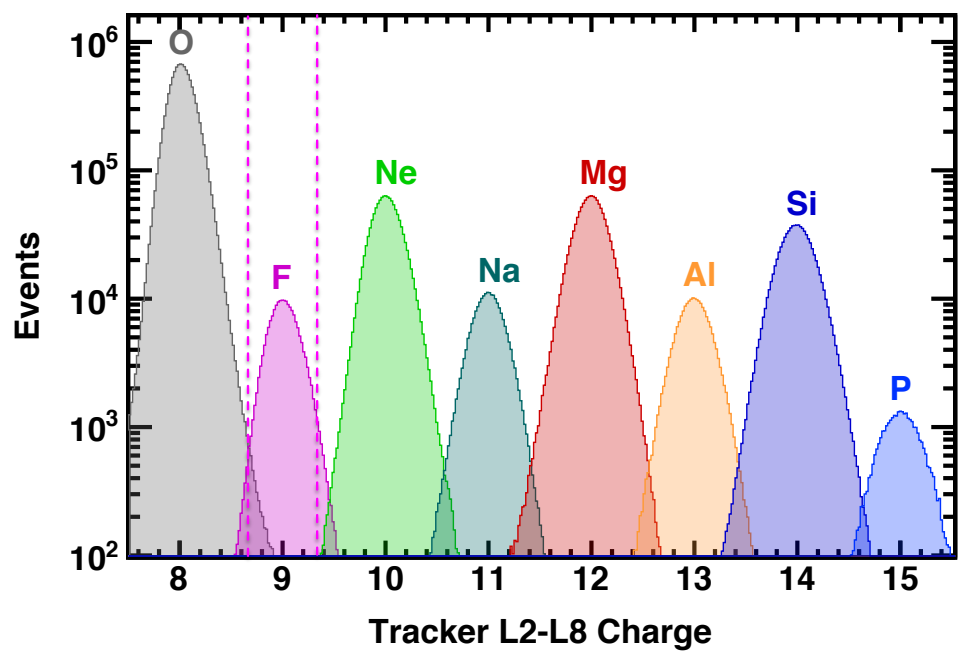

Figure 2: Distributionof the charge measured with the inner tracker $L 2-L 8$ for samples from $Z=8$ to $Z=15$ selected by the combined charge measured with $L 1$, the upper TOF, and the lower TOF over the rigidities above $4 \mathrm{GV}$. The dashed vertical lines correspond to the charge selection in the inner tracker for fluorine.

Charge measurements on $L 1$, the inner tracker, the upper TOF, the lower TOF, and, for $R>1.2$ $\mathrm{TV}, L 9$ are required to be compatible with charge $Z=9$. As an example, Fig. 2 shows the charge measurement for the inner tracker alone. With the selection, the background from charge-adjacent non-interacting nuclei $(\mathrm{O}$ and $\mathrm{Ne}$ ) due to the finite AMS charge resolution is negligible, $<0.5 \%$ over the whole rigidity range. The main background comes from heavier nuclei, such as $\mathrm{Ne}, \mathrm{Mg}$, and $\mathrm{Si}$, which interact above tracker $L 2$. It has two sources. First, the background resulting from interactions in the material between $L 1$ and $L 2$ (TRD and upper TOF) is evaluated by fitting the charge distribution of tracker $L 1$ with charge distribution templates of $\mathrm{O}, \mathrm{F}, \mathrm{Ne}$, and $\mathrm{Na}$. Then cuts are applied on the $L 1$ charge as shown in Fig. 3. The charge distribution templates are obtained using $L 2$. These templates contain only noninteracting events by requiring that $L 1$ and $L 3-L 8$ measure the same charge value. This background varies from $4 \%$ to $15 \%$ depending on rigidity. Second, the background from interactions on materials above $L 1$ (thin support structures made by carbon fiber and aluminum honeycomb) has been estimated from simulation using Monte Carlo 
samples generated according to AMS flux measurements. The simulation of nuclear interactions has been validated with data using nuclear charge changing cross-sections $(\mathrm{Ne}, \mathrm{Mg}, \mathrm{Si}, \ldots \rightarrow \mathrm{F}+\mathrm{X}$ ) [21] measured by AMS. After all backgrounds are subtracted, we obtain $0.29 \times 10^{6}$ fluorine nuclei. The uncertainty due to background subtraction is $1.5 \%$ at $2 \mathrm{GV}, 2 \%$ at $100 \mathrm{GV}$ and $6 \%$ at $2.9 \mathrm{TV}$.

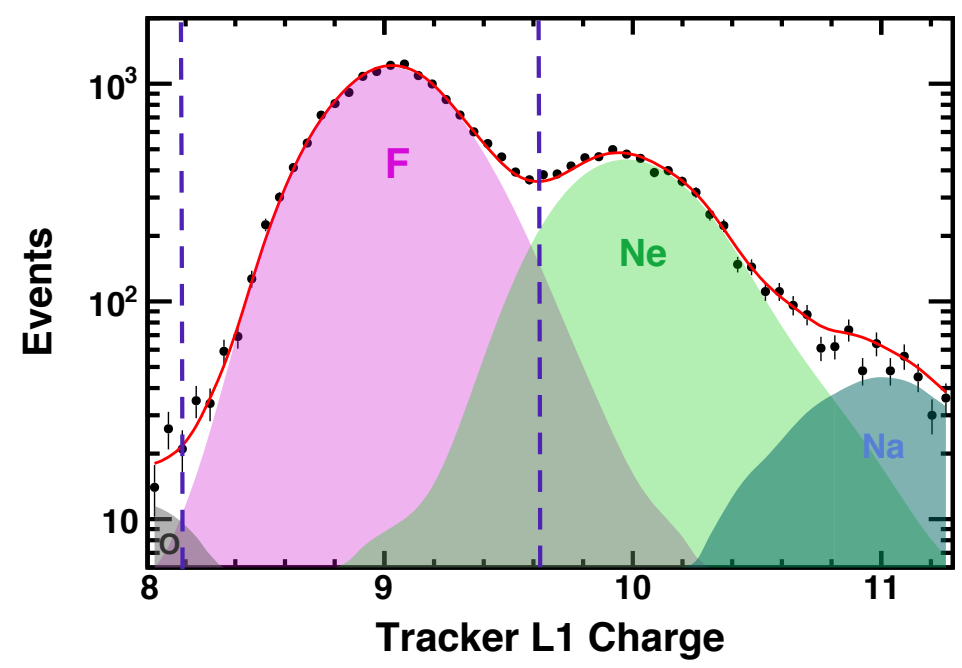

Figure 3: Charge distributions measured by tracker $L 1$ for fluorine events selected by the inner tracker $L 2-L 8$ in the rigidity range between 18 and $22 \mathrm{GV}$ (black dots).

\section{Data Analysis}

The isotropic flux $\Phi_{i}$ in the $i$ th rigidity bin $\left(R_{i}, R_{i}+\Delta R_{i}\right)$ is given by

$$
\Phi_{i}=\frac{N_{i}}{A_{i} \epsilon_{i} T_{i} \Delta R_{i}}
$$

where $N_{i}$ is the number of events corrected for bin-to-bin migration, $A_{i}$ is the effective acceptance, $\epsilon_{i}$ is the trigger efficiency, and $T_{i}$ is the collection time. The flux was measured in 49 bins from $2.15 \mathrm{GV}$ to $2.9 \mathrm{TV}$. The bin-to-bin migration of events was corrected using the unfolding procedure described in Ref. [17].

The systematic error on the fluxes associated with the trigger efficiency measurement is $<1 \%$ over the entire rigidity range.

The effective acceptances $A_{i}$ were calculated using Monte Carlo simulation and corrected for small differences between the data and simulated events related to a) event reconstruction and selection, charge determination, and tracker quality cuts and b) the details of inelastic interactions of nuclei in the AMS materials. The systematic errors on the fluxes associated with the reconstruction and selection are $<1 \%$ over the entire rigidity range.

The material traversed by nuclei from the top of AMS to $L 9$ is composed primarily of carbon and aluminum. The survival probabilities of $\mathrm{F}$ nuclei due to interactions in the materials were evaluated using cosmic ray data collected by AMS as described in Ref. [21]. The systematic error 
due to uncertainties in the evaluation of the inelastic cross section is $<3 \%$ up to $100 \mathrm{GV}$. Above $100 \mathrm{GV}$, the small rigidity dependence of the cross section from the Glauber-Gribov model [20] was treated as an uncertainty and added in quadrature to the uncertainties from the measured interaction probabilities [21]. The corresponding systematic error on the F flux is $<3 \%$ up to $100 \mathrm{GV}$ and rises smoothly to $4 \%$ at $2.9 \mathrm{TV}$.

The rigidity resolution function for $\mathrm{F}$ has been studied following the procedure decribed in Ref. [18]. The resulting systematic error on the flux is less than $1 \%$ below $200 \mathrm{GV}$ and smoothly increasing to $7 \%$ at $2.9 \mathrm{TV}$.

There are two contributions to the systematic uncertainty on the rigidity scale [17]. The first is due to residual tracker misalignment. This error was estimated by comparing the $E / p$ ratio for electrons and positrons, where $E$ is the energy measured with the ECAL and $p$ is the momentum measured with the tracker. It was found to be $1 / 30 \mathrm{TV}^{-1}$ [22]. The second systematic error on the rigidity scale arises from the magnetic field map measurement and its temperature corrections. The error on the F flux due to uncertainty on the rigidity scale is $<1 \%$ up to $200 \mathrm{GV}$ and increases smoothly to $6.5 \%$ at $2.9 \mathrm{TV}$.

\section{Results}

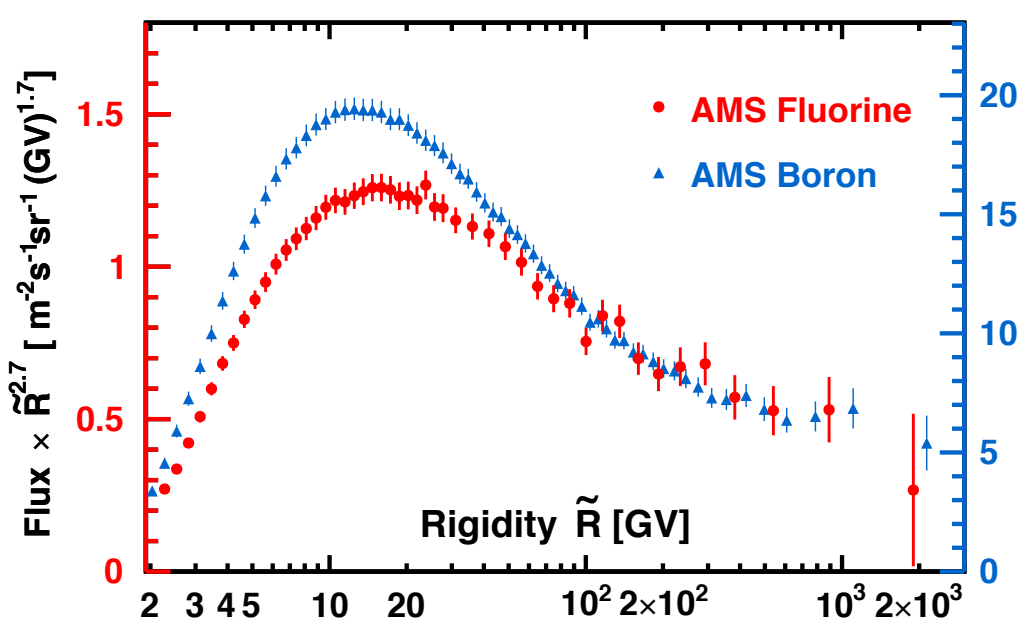

Figure 4: The AMS fluorine F flux multiplied by $\tilde{R}^{2.7}$ with total errors as a function of rigidity (left axis) together with the AMS boron B flux [10] (right axis).

Figure 4 shows measured the $\mathrm{F}$ flux as a function of rigidity $\tilde{R}$ with the total errors, the sum in quadrature of statistical and systematic errors. For comparison Fig. 4a also shows the AMS results on the boron flux [10]. As seen, at high rigidities the rigidity dependences of the F and B fluxes are identical, at low rigidities they are different. Figure 5 shows the AMS fluorine flux as a function of kinetic energy per nucleon $E_{K}$ together with earlier measurements [3-7].

To compare the rigidity dependence of the $\mathrm{F}$ flux with that of the $\mathrm{Ne}, \mathrm{Mg}$, and Si primary cosmic ray fluxes, which have an identical rigidity dependence above $80.5 \mathrm{GV} \mathrm{[11],} \mathrm{the} \mathrm{ratio} \mathrm{of} \mathrm{the}$ F flux to the characteristic heavy primary $\mathrm{Si}$ flux, F/Si, was computed. Figure 6a shows the AMS $\mathrm{F} / \mathrm{Si}$ flux ratio as a function of rigidity together with the AMS B/O flux ratio [10]. 


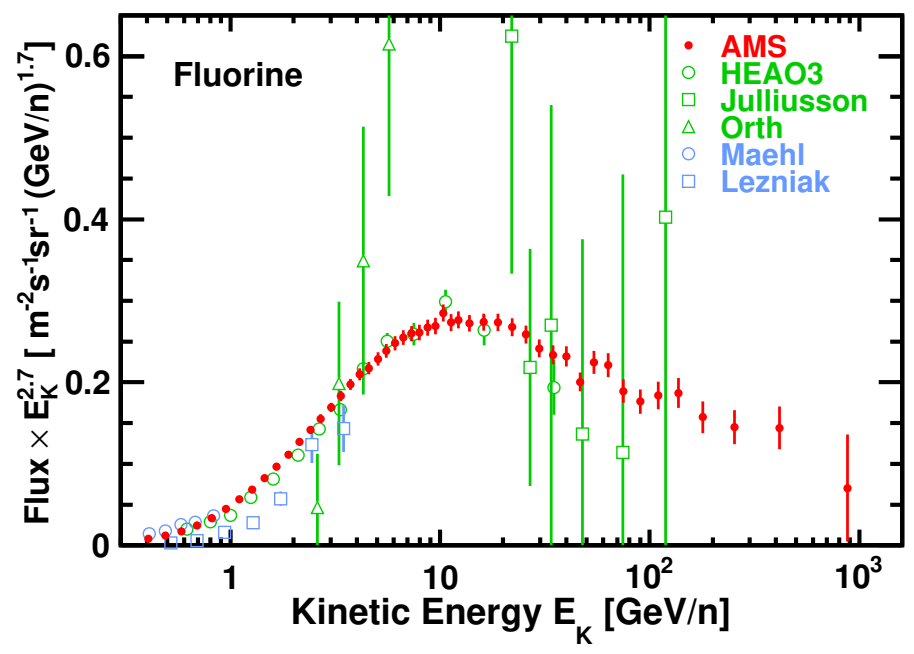

Figure 5: The AMS fluorine flux as a function of kinetic energy per nucleon $E_{K}$ multiplied by $E_{K}^{2.7}$ together with earlier measurements [3-7].

The variation with rigidity of the spectral index $\Delta$ of F/Si flux ratio was obtained by fitting it with

$$
\begin{cases}C(R / 175 \mathrm{GV})^{\Delta_{1}}, & R \leq 175 \mathrm{GV}, \\ C(R / 175 \mathrm{GV})^{\Delta_{2}}, & R>175 \mathrm{GV} .\end{cases}
$$

over rigidity interval [28.8-2900] GV. The fit yields $C^{\mathrm{F} / \mathrm{Si}}=0.0044 \pm 0.0001, \Delta_{1}^{\mathrm{F} / \mathrm{Si}}=-0.34 \pm 0.02$, and $\Delta_{2}^{\mathrm{F} / \mathrm{Si}}=-0.19 \pm 0.07$ with $\chi^{2} /$ d.o.f. $=13 / 16$. Above $175 \mathrm{GV}$ the spectral index $\Delta^{\mathrm{F} / \mathrm{Si}}$ exhibits a hardening $\left(\Delta_{2}^{\mathrm{F} / \mathrm{Si}}-\Delta_{1}^{\mathrm{F} / \mathrm{Si}}\right)$ of $0.15 \pm 0.07$, compatible with $\mathrm{AMS}$ results on the $\mathrm{Li} / \mathrm{C}, \mathrm{Be} / \mathrm{C}, \mathrm{B} / \mathrm{C}$, $\mathrm{Li} / \mathrm{O}, \mathrm{Be} / \mathrm{O}$, and $\mathrm{B} / \mathrm{O}$ flux ratios hardening of $0.140 \pm 0.025[9,10]$. Figure $6 \mathrm{a}$ also shows the AMS F/Si fit results with Eq. (2) together with the predictions of the cosmic ray propagation model GALPROP [23] and of the latest GALPROP-HELMOD model [2] on the F/Si flux ratio, and the AMS B/O fit results with Eq. (2), $C^{\mathrm{B} / \mathrm{O}}=0.097 \pm 0.003, \Delta_{1}^{\mathrm{B} / \mathrm{O}}=-0.405 \pm 0.005$, and $\Delta_{2}^{\mathrm{B} / \mathrm{O}}=-0.26 \pm 0.03$ with $\chi^{2} /$ d.o.f. $=24 / 36$.

To compare the rigidity dependence of the $\mathrm{F} / \mathrm{Si}$ flux ratio with the lighter secondary-to-primary $\mathrm{B} / \mathrm{O}$ flux ratio in detail, the $\frac{\mathrm{F} / \mathrm{Si}}{\mathrm{B} / \mathrm{O}}$ ratio was computed and shown in Fig. $6 \mathrm{~b}$. Over the entire rigidity range $\frac{\mathrm{F} / \mathrm{Si}}{\mathrm{B} / \mathrm{O}}$ can be fitted with

$$
\frac{\mathrm{F} / \mathrm{Si}}{\mathrm{B} / \mathrm{O}}= \begin{cases}k\left(R / R_{0}\right)^{\delta_{l}}, & R \leq R_{0}, \\ k\left(R / R_{0}\right)^{\delta}, & R>R_{0} .\end{cases}
$$

The fit yields $k=0.39 \pm 0.01, R_{0}=9.8 \pm 0.9 \mathrm{GV}, \delta_{l}=-0.055 \pm 0.013$, and $\delta=0.052 \pm 0.007$ with $\chi^{2} /$ d.o.f. $=24 / 45$. As seen, the rigidity dependence of the $\mathrm{F} / \mathrm{Si}$ and $\mathrm{B} / \mathrm{O}$ flux ratios are distinctly different. Most importantly, the latest AMS result shows that above $10 \mathrm{GV}$ the $\frac{\mathrm{F} / \mathrm{Si}}{\mathrm{B} / \mathrm{O}}$ ratio can be described by a single power law $\propto R^{\delta}$ with $\delta=0.052 \pm 0.007$ (a $7 \sigma$ difference from zero). This shows, unexpectedly, that the heavier secondary-to-primary F/Si flux ratio rigidity dependence is distinctly different from the lighter $\mathrm{B} / \mathrm{O}$ (or $\mathrm{B} / \mathrm{C}$ ) rigidity dependence, indicating that the propagation 
properties of heavy cosmic rays, from $\mathrm{F}$ to $\mathrm{Si}$, are different from those of light cosmic rays, from He to $\mathrm{O}$.
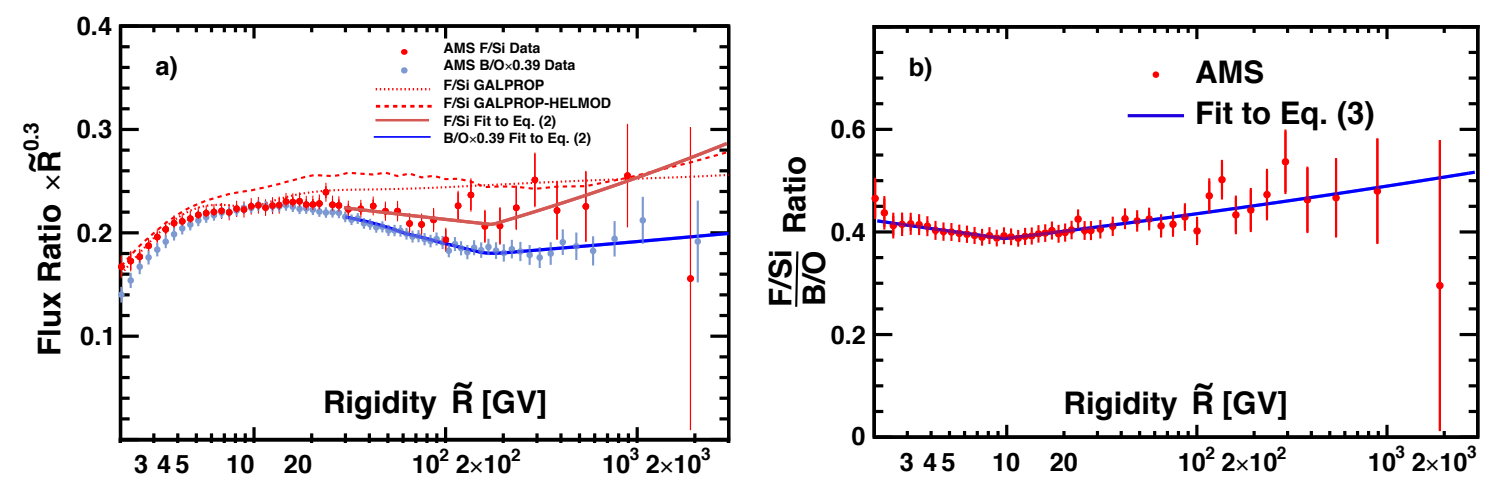

Figure 6: a) The AMS F/Si flux ratio (red dots) and B/O flux ratio (blue dots) as a function of rigidity with total errors. For display purposes only, the $\mathrm{F} / \mathrm{Si}$ and $\mathrm{B} / \mathrm{O}$ flux ratios are multiplied by $\tilde{R}^{0.3}$ and the $\mathrm{B} / \mathrm{O}$ flux ratio rescaled as indicated. b) The AMS $\frac{\mathrm{F} / \mathrm{Si}}{\mathrm{B} / \mathrm{O}}$ ratio as a function of rigidity with total errors.

\section{Conclusions}

We have presented the precision measurement of the $\mathrm{F}$ flux as a function of rigidity from $2.15 \mathrm{GV}$ to $2.9 \mathrm{TV}$, with detailed studies of the systematic errors. The fluorine spectrum deviates from a single power law above $200 \mathrm{GV}$. The heavier secondary-to-primary F/Si flux ratio rigidity dependence is distinctly different from the lighter B/O (or B/C) rigidity dependence. In particular, above $10 \mathrm{GV}$, the $\frac{\mathrm{F} / \mathrm{Si}}{\mathrm{B} / \mathrm{O}}$ ratio can be described by a power law $R^{\delta}$ with $\delta=0.052 \pm 0.007$, revealing that the propagation properties of heavy cosmic rays, from $\mathrm{F}$ to $\mathrm{Si}$, are different from those of light cosmic rays, from $\mathrm{He}$ to $\mathrm{O}$.

\section{Acknowledgements}

We acknowledge the support of the DOE including resources from the National Energy Research Scientific Computing Center under Contract No. DE-AC02-05CH11231 and the Argonne Leadership Computing Facility under Contract No. DE-AC02-06CH11357. We are grateful for the continuous support from MIT and its School of Science. We thank the strong support from CERN IT department.

\section{References}

[1] I. A. Grenier, J. H. Black, and A. W. Strong, Annu. Rev. Astron. Astrophys. 53, 199 (2015); P. Blasi, Astron. Astrophys. Rev. 21, 70 (2013); A. W. Strong, I. V. Moskalenko, and V. S. Ptuskin, Annu. Rev. Nucl. Part. Sci. 57, 285 (2007); A. Castellina and F. Donato, Astropart. Phys. 24, 146 (2005).

[2] M. J. Boschini et al., Astrophys. J. Supp. Series, 250, 1 (2020). 
[3] E. Juliusson, Astrophys. J. 191, 331 (1974).

[4] R. Maehl et al., Astrophys. J. Supp. Series, 47, 163 (1977).

[5] C. Orth et al., Astrophys. J. 226, 1147 (1978).

[6] J. Lezniak and W. Webber, Astrophys. J. 223, 676 (1978).

[7] J. Engelmann et al., Astron. Astrophys. 233, 96 (1990).

[8] C. Evoli et al., Phys. Rev. D 99103023 (2019).

[9] M. Aguilar et al., Phys. Rev. Lett. 120, 021101 (2018).

[10] M. Aguilar et al., "The Alpha Magnetic Spectrometer (AMS) on the international space station: Part II - Results from the first seven years", Physics Reports, in press.

[11] M. Aguilar et al., Phys. Rev. Lett. 124, 211102 (2020).

[12] A. Kounine, Int. J. Mod. Phys. E 211230005 (2012); S. Ting, Nucl. Phys. B, Proc. Suppl. 243-244, 12 (2013); M. Incagli, AIP Conf. Proc. 1223, 43 (2010); R. Battiston, Nucl. Instrum. Methods Phys. Res., Sect. A 588, 227 (2008).

[13] B. Alpat et al., Nucl. Instrum. Methods Phys. Res., Sect. A 613, 207 (2010).

[14] Y. Jia, Q. Yan, V. Choutko, H. Liu, and A. Oliva, Nucl. Instrum. Methods Phys. Res., Sect. A 972, (2020).

[15] G. Ambrosi, V. Choutko, C. Delgado, A. Oliva, Q. Yan, and Y. Li, Nucl. Instrum. Methods Phys. Res., Sect. A 869, 29 (2017).

[16] V. Bindi et al., Nucl. Instrum. Methods Phys. Res., Sect. A 743, 22 (2014).

[17] M. Aguilar et al., Phys. Rev. Lett. 114, 171103 (2015).

[18] M. Aguilar et al., Phys. Rev. Lett. 115, 211101 (2015).

[19] C. C. Finlay et al., Geophys. J. Int. 183, 1216 (2010); E. Thébault et al., Earth Planets Space 67, 79 (2015); Geomagnetic Field Modeling Working Group, IGRF-13 model, 2019.

[20] J. Allison et al., Nucl. Instrum. Methods Phys. Res., Sect. A 835, 186 (2016); J. Allison et al., IEEE Trans. Nucl. Sci. 53, 270 (2006); S. Agostinelli et al., Nucl. Instrum. Methods Phys. Res., Sect. A 506, 250 (2003).

[21] Q. Yan, V. Choutko, A. Oliva, and M. Paniccia, Nuclear Physics A 996121712 (2020).

[22] J. Berdugo, V. Choutko, C. Delgado, and Q. Yan, Nucl. Instrum. Methods Phys. Res., Sect. A 869, 10 (2017).

[23] We used GALPROP WebRun, A. E. Vladimirov, S. W. Digela, G. Jóhannesson, P. F. Michelson, I. V. Moskalenko, P. L. Nolan, E. Orlando, T. A. Porter, and A. W. Strong, Comput. Phys. Comm. 182, 1156 (2011), with parametrization from R. Trotta, G. Jóhannesson, I. V. Moskalenko, T. A. Porter, R. Ruiz de Austri, and A. W. Strong, Astrophys. J. 729, 106 (2011). 